
\title{
GMecolocical cinicer Role and impact of multimodal prehabilitation for gynecologic oncology patients in an Enhanced Recovery After Surgery (ERAS) program
}

Ester Miralpeix, ${ }^{01}$ Gemma Mancebo, ${ }^{1}$ Sonia Gayete, ${ }^{1}$ Marta Corcoy, ${ }^{2}$ Josep-Maria Solé-Sedeño ${ }^{1}$

${ }^{1}$ Department of Obstetrics ad Gynecology, Hospital del Mar, Barcelona, Spain

${ }^{2}$ Department of Anesthesia, Hospital del Mar, Barcelona, Spain

\section{Correspondence to}

Mrs Ester Miralpeix,

Department of Obstetrics and Gynecology, Hospital del Mar, Barcelona E-08003, Spain; ester.miralpeix@gmail.com

Received 6 May 2019 Revised 15 July 2019 Accepted 16 July 2019 Published Online First 30 August 2019
Check for updates

(C) IGCS and ESGO 2019. № commercial re-use. See rights and permissions. Published by BMJ.

To cite: Miralpeix E, Mancebo G, Gayete S, et al. Int J Gynecol Cancer 2019;29:1235-1243.

\begin{abstract}
Patients undergoing major surgery are predisposed to a decrease in functional capacity as a response to surgical stress that can delay post-operative recovery. A prehabilitation program consists of patient preparation strategies before surgery, and include pre-operative measures to improve functional capacity and enhance post-operative recovery. Multimodal prehabilitation may include exercise, nutritional counseling, psychological support, and optimization of underlying medical conditions, as well as cessation of unfavorable health behaviors such as smoking and drinking. Currently, there are no standardized guidelines for prehabilitation, and the existent studies are heterogeneous; however, multimodal approaches are likely to have a greater impact on functional outcomes than single management programs. We have reviewed the literature on prehabilitation in general, and in gynecologic surgery in particular, to identify tools to establish an optimal prehabilitation program within an Enhanced Recovery After Surgery (ERAS) protocol for gynecologic oncology patients. We suggest a safe, reproducible, functional, and easy-to-apply multimodal prehabilitation program for gynecologic oncology practice based on patienttailored pre-operative medical optimization, physical training, nutritional counseling, and psychological support. The analysis of the prehabilitation program implementation in an ERAS protocol should undergo further research in order to test the efficacy on surgical outcome and recovery after surgery.
\end{abstract}

\section{INTRODUCTION}

Surgery disrupts the physiologic balance and triggers a general stress response, altering hormonal, metabolic, immunologic, and neurological functions. ${ }^{1}$ Even without peri-operative complications, surgical stress is associated with a decrease of $20-40 \%$ of functional capacity. ${ }^{2}$ In addition, a reduction of surgical stress may be considered a key factor for early recovery after surgery. The fasttrack surgery (FTS) or Enhanced Recovery After Surgery (ERAS) are multimodal peri-operative care pathways that maintain physiological normality and reduce surgical stress to accelerate post-operative recovery. ${ }^{34}$ ERAS programs in gynecologic oncology have been introduced during the last several years, and guidelines for optimal peri-operative care of gynecologic oncology patients have been updated in $2019 .{ }^{56}$

A step forward in peri-operative care is offered by the period between diagnosis and the surgical procedure, which offers an ideal opportunity to optimize patient status. Prehabilitation programs are aimed at enhancing surgical recovery by raising the patient's functional and metabolic reserves before intervention. Since surgery has an impact on the physical, psychological, and nutritional status of patients, multimodal prehabilitation programs assess individual functional capacity through physical, nutritional, and emotional tests. Multimodal prehabilitation programs may include exercise, nutritional counseling (eg, protein supplementation), psychological support (eg, stress-reducing strategies), and strategies to optimize underlying conditions and promote cessation of negative health behaviors. ${ }^{17}$ Moreover, medical optimization during the pre-operative period is necessary to identify comorbidities such as hypertension and diabetes, and to improve potentially modifiable risk factors such as fragility, anemia, smoking and/or alcohol intake, which are strongly associated with post-operative complications. ${ }^{8}$

It seems reasonable to assume that combining the pre-operative optimization provided by a prehabilitation program with the reduction of surgical stress provided by an ERAS program could further improve post-surgical recovery. However, studies that analyze the effect of prehabilitation added to ERAS peri-operative care on surgical recovery are still scarce ${ }^{9-11}$ especially in the gynecological field.

Thus, this study aims to review the current evidence on prehabilitation programs and the impact of such programs on recovery outcomes, as well as to identify key elements in establishing a successful multimodal prehabilitation program combined with an ERAS surgical approach in gynecologic oncology patients. As a result, we ultimately propose a prehabilitation protocol that is currently ongoing in our department. 


\section{Review Article}

\section{METHODS}

In this study we searched the Medline, EMBASE, PubMed, and Cochrane Library databases for articles published in English with the following keywords: prehabilitation, and rehabilitation. These were combined with the keywords cancer, surgery, gynecologic surgery, gynecologic oncology, and outcomes. Our search was limited to the period from January 2000 to April 2018, since the initial publications that met our inclusion criteria were inclusive in those time points.

From the articles identified, we selected both randomized controlled trials as well as observational studies that reported on any of the following outcome parameters in the context of an established prehabilitation program: length of hospital stay, post-operative pain and analgesia, peri-operative complications, readmission rates, time to return to daily activities, quality of life, and cost analysis. We excluded case reports and reports that mentioned a non-standardized parameter for prehabilitation evaluation. From all included studies, we reviewed the key elements, such as procedures and compliance, and evaluated their impact on recovery after surgery.

\section{RESULTS}

\section{Prehabilitation in General Surgery}

Pre-operative poor functional capacity is a risk factor for higher morbidity and mortality after major surgery. ${ }^{12}$ Prehabilitation before abdominal or cardiac surgery improves post-operative recovery and reduces post-operative complications and length of stay in elderly patients. ${ }^{13}$ Until recently, prehabilitation programs were mainly directed towards physical interventions, such as physical training. However, nutritional and psychological interventions have also shown a beneficial effect on post-operative recovery, and their inclusion in multimodal prehabilitation programs may increase the benefits of physical training alone. ${ }^{1415}$ Thus, we reviewed the literature on the physical, nutritional, and psychological aspects included in prehabilitation programs.

\section{Physical Intervention}

Patients with adequate pre-operative physical activity and inspiratory muscle strength had better post-operative outcomes and shorter length of hospital stay. ${ }^{16}$ Eight studies (five randomized controlled, one systematic review, one meta-analysis, and two cohort studies) were identified for physical interventions before surgery and are summarized here. A systematic review that assessed the effect of pre-operative physical fitness on post-operative outcomes after abdominal surgery showed that prehabilitation consisting of inspiratory muscle training, aerobic exercise, and/ or resistance training decreases post-operative complications after abdominal surgery (odds ratio $0.59,95 \%$ confidence interval (Cl) 0.38 to $0.91 ; p=0.03) .{ }^{17}$ Therefore, physical training (ie, exercise and inspiratory muscle training) is the main target of most prehabilitation programs.

Functional capacity is commonly evaluated by cardiopulmonary exercise testing; however, this requires significant resources including equipment, personnel, and expertise. ${ }^{18}$ Alternatively, exercise capacity is evaluated by the 6-min walk test (6-MWT), which is a reproducible test that has been validated in surgical populations. 6-MWT is associated with the capacity to perform daily activities, and registers the maximum distance a patient is able to walk during 6 min at moderate intensity. ${ }^{19}{ }^{20}$ This test is linearly correlated to maximum oxygen consumption ( $\mathrm{V}_{2} \max$ ) that is associated with post-operative morbidity and complications. ${ }^{21}$ Patients who walked $<350$ meters experienced more short-term and long-term morbidity and higher mortality. ${ }^{22}$

There is large heterogeneity according to type, intensity, duration, timing, and supervision of exercise interventions in prehabilitation programs; however, most have been proved to improve recovery after surgery and reduce post-operative complications. Soares et al compared 16 patients undergoing upper abdominal surgery, assigned to receive two supervised and four unsupervised exercise sessions for 2-3 weeks pre-operatively, to 16 control patients without physical therapy. Patients in the intervention group had higher inspiratory capacity before surgery and 6-MWT on post-operative day $7(p<0.05)$. Moreover, post-operative complication rates were reduced in the intervention group $(31.3 \%$ vs $68.7 \% ; p=0.03){ }^{23}$ Barberan-Garcia et al developed a personalized exercise prehabilitation program in high-risk patients undergoing major abdominal surgery. This involved one to three supervised sessions per week based on a cycle-ergometer stationary bicycle combined with a daily unsupervised personalized program. The prehabilitation group achieved better aerobic capacity during the pre-operative period $(p<0.001)$ and the rate of post-operative complications decreased $(31 \%$ vs $62 \% ; p=0.001) .{ }^{15}$ In colorectal surgery, Carli et al compared 58 patients randomized to a daily unsupervised structured cycling and strengthening program, to a control group with an unsupervised daily walking and breathing program (54 patients). There were no differences between the groups in mean functional walking capacity over the prehabilitation period or at post-operative follow-up. In fact, post-operative improvement was greater in the control group ( $41 \%$ vs $11 \% ; p=0.019)$. Nevertheless, a compliance rate of only $16 \%$ was reported. ${ }^{24}$ Gillis et al compared a prehabilitation group based on home-based unsupervised exercise for at least 3 days per week before surgery with a rehabilitation group that initiated exercise at home after surgery. Although the mean baseline walking capacity measured by 6-MWT was the same between groups, functional walking capacity increased in a higher proportion in the prehabilitation group compared with the rehabilitation group ( $53 \%$ vs $15 \%, p=0.006 \%$, and $84 \%$ vs $62 \%$, $\mathrm{p}=0.049$, respectively) over the peri-operative time and 8 weeks after surgery. Complication rates and length of hospital stay were similar between groups. ${ }^{25}$

Physical intervention in prehabilitation programs may also include inspiratory muscle training. Dronkers et al reported an association between adequate pre-operative inspiratory muscle training and shorter length of hospital stay after major oncological abdominal surgery. ${ }^{16}$ In elective cardiac surgery, pre-operative inspiratory muscle training reduces post-operative pulmonary complications. ${ }^{26}$ Similar effects are suggested for thoracic, abdominal, and orthopedic surgery. ${ }^{27}$ Inspiratory muscle training involves 15 to $30 \mathrm{~min}$ daily sessions using an inspiratory threshold-loading device with a patient-tailored program or daily home breathing exercises through a flow volume incentive spirometer. ${ }^{28}$

Lastly, physical intervention can be home-based or can be performed under the supervision of a healthcare provider. Although both types of training have been reported to be beneficial, 
supervised structured exercises encourage patient compliance and enhance functional capacity after surgery. ${ }^{29}$

\section{Nutritional Intervention}

Regardless of baseline nutritional status, surgical trauma induces a stress response that increases oxygen consumption, mobilizes energy reserves, and promotes protein catabolism, ultimately resulting in skeletal muscle wasting. ${ }^{1}$ Therefore, ERAS programs recommend pre-operative carbohydrate loading to minimize the catabolic influence of the surgical stress response. ${ }^{6}$

Five studies (three randomized controlled trials, one systematic review, and one cohort study) were identified for physical interventions before surgery in our literature review and are summarized in this section. A recent Cochrane systematic review concluded that pre-operative carbohydrate treatment compared with placebo or fasting reduces the length of hospital stay $(0.30$ days, $95 \% \mathrm{Cl} 0.56$ to 0.04 ) in patients undergoing elective surgery (abdominal, orthopedic, cardiac, and thyroidectomy), although it has no significant effect on post-operative complication rates. ${ }^{30}$

Patients who are malnourished pre-operatively have a greater risk of morbidity and mortality and prolonged hospital stay. ${ }^{31}$ Nutritional intervention before surgery is particularly relevant in those patients. Importantly, malnutrition and cachexia are common in patients undergoing cancer surgery due to neoplastic disease, chronic inflammatory state, alimentary tract dysfunction, or psychological disorders such as low mood and stress-related anorexia. ${ }^{32}$ Jie et al published an observational study showing that post-operative complication rates before abdominal surgery decreased in malnourished patients who received adequate pre-operative nutrition. ${ }^{33}$ Studies in patients with colorectal cancer resection showed no significant beneficial reduction in post-operative complications following nutritional supplementation, ${ }^{11}{ }^{34}$ although patients who received whey protein supplementation 4 weeks before surgery had a mean improvement in functional walking capacity. ${ }^{11} \mathrm{~A}$ prospective randomized study in non-malnourished patients undergoing abdominal cancer surgery showed that patients who received nutritional supplementation for 14 days before surgery significantly reduced the number and severity of post-operative complications. Moreover, laboratory parameters decreased in the control group, while in the nutritional supplementation group they were stable (albumin and total protein) or raised (transferrin and total lymphocyte count) after surgery. ${ }^{35}$

Although nutritional supplementation could be beneficial for all surgical patients, malnourished patients should be identified early during pre-operative evaluation to enable appropriate nutritional intervention. To this end, there are many nutrition screening tools designed to detect malnutrition, such as the Malnutrition Universal Screening Tool (MUST), Nutrition Risk Index, and Nutrition Risk Screening. MUST is described as a simple and quick method that is easy to use by healthcare professionals, with high validity for early detection of malnutrition and guide for intervention. ${ }^{36}$

Lastly, it is important to take into account that nutritional supplements act synergistically with exercise by allowing successful muscle gain during physical exercise and optimizing its effect. ${ }^{37}$

\section{Psychological Intervention}

The pre-operative period is an uncertain time for patients, who usually experience anxiety for a variety of reasons including diagnosis (ie, cancer), surgery, and possible complications. Six studies (three randomized controlled trials, two systematic reviews, and one cohort study) were identified for psychological interventions before surgery and are summarized in this section.

Patients who present with pre-operative psychological distress may have a worse surgical recovery and a higher risk of mortality. ${ }^{3839}$ In agreement with that, some prehabilitation programs include psychological interventions before surgery. The psychological status of patients is usually assessed with the Hospital Anxiety and Depression Scale (HADS). ${ }^{40}$

The effect of psychological intervention in a prehabilitation program is controversial. Barberan-Garcia et al evaluated baseline and pre-operative HADS scores in a randomized blinded controlled trial of patients undergoing major abdominal surgery without improvement in psychological status. The intervention group underwent a personalized prehabilitation program based on motivational interview and physical activity, with absence of a specific psychological intervention. ${ }^{15}$ Another randomized study in patients undergoing colorectal resection for cancer compared a 4-week trimodal prehabilitation program to a control group (which received the same interventions but only immediately after surgery), and found no significant differences in the HADS score. The psychological intervention consisted of training by a psychologist on relaxation exercises that could be performed at home. ${ }^{25}$

In contrast, a similar psychological intervention significantly reduced the HADS score during the pre-operative period of time, although without reaching a clinically relevant difference. In this study, the control group was assessed only immediately before surgery, so changes during the pre-operative period could not be evaluated. ${ }^{7}$ Moreover, a Cochrane review about the effect of psychological intervention on post-operative outcomes suggested that emotional support may be beneficial for post-operative pain, behavioral recovery, and length of stay. ${ }^{41}$ Thus, psychological intervention in a prehabilitation program could be important to improve surgical recovery. Nevertheless, its effect on prehabilitation programs needs further assessment to identify which method is more effective and to promote best compliance.

Lastly, it is important to consider that the addition of a psychological intervention in a prehabilitation program could also help to reinforce patient motivation to follow physical exercise and nutritional interventions. Conversely, physical exercise has been associated with an improvement of anxiety and depressive symptoms. ${ }^{42}$ Moreover, although anxious patients showed a greater improvement in functional capacity during the prehabilitation period, they were ultimately less likely to recover to baseline after surgery. ${ }^{43}$

\section{Multimodal Prehabilitation Programs}

As previously stated, surgery has an impact on physical capacity, but also on nutritional and emotional status. ${ }^{17}$ Emerging evidence suggests that a prehabilitation program adding nutritional and psychological strategies to physical intervention has a better impact on functional outcomes, when compared with a single prehabilitation modality. ${ }^{14}$ Four studies (one randomized controlled trial, two systematic reviews, and one cohort study) were identified for multimodal prehabilitation programs and are discussed in this section.

In 2013, Li et al ${ }^{7}$ published a pilot study with a 4-week trimodal prehabilitation program that consisted of moderate exercise, protein supplementation, and anxiety-reduction interventions in 


\section{Review Article}

patients scheduled for colorectal cancer resection. At 8 weeks post-surgery, $81 \%$ of patients who had prehabilitation recovered baseline functional capacity, compared with $40 \%$ of patients in the control group $(p<0.01)$. Importantly, similar results were found in a randomized controlled trial conducted by Gillis et al. ${ }^{25}$ This multimodal prehabilitation program consisted of a home-based, unsupervised, exercise program plus nutritional supplementation and anxiety reduction techniques. The program was initiated 4 weeks before surgery or immediately after surgery, and continued for 8 weeks into the post-operative period. In agreement with the results of $L i$ et al, a higher proportion of patients in the prehabilitation group recovered baseline exercise capacity at 8 weeks compared with the control group ( $84 \%$ vs $62 \% ; p=0.049$ ). Recently, data from these two studies and a new randomized control trial have been pooled and analyzed. Combined analysis of data from 185 patients showed that trimodal prehabilitation improves pre-operative physical fitness and postoperative 6-MWT at 4 and 8 weeks after surgery compared with a rehabilitation program alone. ${ }^{44}$

The mean duration of the prehabilitation programs is heterogeneous and ranges between 2-6 weeks pre-operatively and 4-8 weeks post-operatively. ${ }^{15}$ 23-34 However, there are no reports available that reflect an association between the length time of the prehabilitation program and outcomes. The duration of the prehabilitation program depends on the time interval before the surgical date, patient status, and type of disease.

Overall, studies are heterogeneous and high-level evidence to support a benefit of these interventions is still lacking. Furthermore, standardization is required in order to compare different interventions.

\section{Prehabilitation in Gynecological Surgery}

There are few studies published on prehabilitation programs in gynecologic surgery, and even fewer in gynecologic oncology patients. ${ }^{45}$ Carli et al provided a detailed prehabilitation strategy on a frail elderly patient with endometrial cancer who underwent robotic-assisted total hysterectomy. The patient underwent physical, nutritional, and psychological assessments before a 3-week prehabilitation home-based program consisting of exercise and nutritional optimization. The patient was followed within 8 weeks post-surgery, showing sustained improvement in exercise tolerance, cognitive function, and overall functional capacity. ${ }^{46}$ Another prospective, multi-center, single-blinded, randomized trial by Vonk Noordegraaf et al included 215 patients who underwent hysterectomy or laparoscopic surgery for benign adnexal masses. The intervention group received personal pre- and post-surgical recommendations for daily activity, work, and self-empowerment. This program had a significant benefit on time to return to work, pain intensity, and quality of life for the intervention group; however, the authors did not provide detailed information on the physical, nutritional, and psychological status of the patients. ${ }^{47}$ In the field of gynecologic oncology, Hawkes et al provided an ongoing, multicenter, three-armed, randomized, phase II trial for obese patients with early stage of endometrial cancer to investigate the effectiveness of conservative treatment options. The proposed study focused on the weight loss intervention along with anti-hormonal treatment (levonorgestrel intra-uterine device) with or without metformin. The authors suggest that the results of this trial could benefit patients and reduce health costs through a reduction in hospitalizations and a lower incidence of adverse events currently observed with standard treatment. ${ }^{48}$

Unlike other disciplines in which prehabilitation has been wellstudied through a well-structured multimodal prehabilitation program, a gynecologic focus still needs to be established and further studies are required to clarify the benefits for these patients.

\section{Design of a Multimodal Prehabilitation Program for Gynecologic Oncology}

Based on the published literature, and considering pre-operative time as a window of opportunity for prehabilitation, we propose a multimodal prehabilitation program for patients undergoing elective gynecologic oncology surgery. It includes: medical optimization, physical intervention, nutritional counseling, and psychological support. In addition, peri-operative care follows the guidelines of the ERAS program, ${ }^{6}$ which has been the standard of surgical approach for our patients since 2014.

Patients with an indication for elective gynecologic oncology surgery are eligible for the prehabilitation program by surgeons and anesthesiologists during consultation. Patients are excluded if $<18$ years of age. Patients must sign an informed consent at the pre-operative evaluation to be included in the program.

Once recruited, all patients are required to attend three evaluation visits: at the time of diagnosis (baseline), a week before surgery (pre-operative), and 8 weeks after surgery (post-operative). The baseline assessment is performed approximately 2 to 4 weeks before the scheduled surgery date, while patients are undergoing complementary pre-operative tests (ie, computed tomography scan or magnetic resonance imaging). In the baseline assessment, we first identify pre-operative status assessing nutritional, physical, and psychological factors, and we prescribe either a home-based program or a supervised program, depending on the results of the screening tests. During the week before surgery (pre-operative) and 8 weeks after surgery (post-operative), we assess the patients' compliance through their daily diaries, and re-evaluate their physical, nutritional, and emotional status. During the peri-operative time all patients are enrolled in an ERAS program. Patients restart their multimodal prehabilitation immediately after surgery (during their hospital stay), and continue it following discharge for up to 8 weeks.

All patients receive an extensive education session over the interventions of the program during their consultation with the surgeon and with the anesthesiologist. Moreover, all patients are provided with an information booklet containing instructions about physical activity, protein supplementation, and relaxation techniques. The booklet also includes a diary where patients are required to record all activities. Based on the patient's diary information, compliance is evaluated. Finally, all patients are administered the Short Form 12-item Survey (SF-12) questionnaire to evaluate their general health status at baseline, before surgery, and 8 weeks after surgery. ${ }^{49}$ Although the recommended length for a prehabilitation period is $2-4$ weeks before surgery, the length of our program is not fixed and depends on the patient's status, tumor type, and extension.

Gynecologic cancer includes a heterogeneous group of patients and diseases, so we adapt and individualize the program according to the patient's functional status, comorbidities, and cancer type. For endometrial and cervical cancer patients, the length of the 


\begin{tabular}{|c|c|c|c|c|}
\hline PREOPERATIVE & \multirow[b]{2}{*}{ 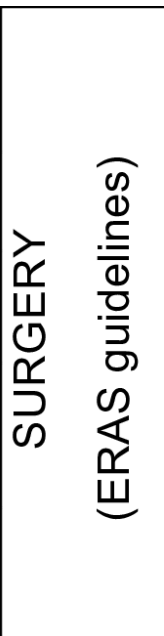 } & $\begin{array}{l}\text { POSTOPERATIVE } \\
\text { (in-hospital stay) }\end{array}$ & \multirow[b]{2}{*}{ 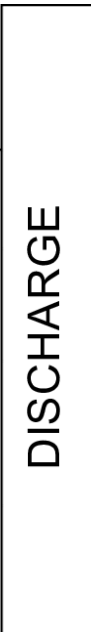 } & POSTOPERATIVE \\
\hline $\begin{array}{l}\text { Medical optimization } \\
\text { Physical intervention } \\
\text { Nutritional } \\
\text { intervention } \\
\text { Psychological } \\
\text { intervention }\end{array}$ & & $\begin{array}{l}\text { Physical exercise } \\
\text { Nutritional support } \\
\text { Psychological support } \\
\text { (if necessary) }\end{array}$ & & $\begin{array}{l}\text { Physical exercise } \\
\text { Nutritional support } \\
\text { Psychological support } \\
\text { (if necessary) }\end{array}$ \\
\hline 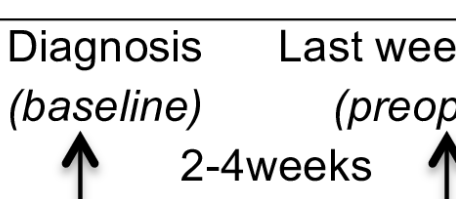 & $\begin{array}{l}\text { before } \\
\text { rative) }\end{array}$ & urgery & & $\begin{array}{c}8 \text { weeks } \\
\text { (postoperative) } \\
\uparrow\end{array}$ \\
\hline
\end{tabular}

\section{Time of prehabilitation intervention assessment}

Figure 1 Study design of the prehabilitation program combined with an ERAS approach for gynecology oncologic patients. ERAS, Enhanced Recovery After Surgery.

prehabilitation program ranges between $2-4$ weeks, a period between diagnosis and surgery where the patients perform complementary pre-operative and imaging tests. For ovarian cancer, all patients start prehabilitation when the disease is suspected or diagnosed, and the length of the program varies according to the treatment approach. In the case of primary debulking surgery, patients usually undergo 2 weeks of prehabilitation, between baseline assessments, laparoscopic evaluation of disease extent, and primary surgery. In the case of interval debulking surgery, patients undergo prehabilitation during neoadjuvant chemotherapy treatment, which usually lasts 2 months. Figure 1 shows the design of our multimodal prehabilitation program for patients undergoing elective gynecologic oncology surgery.
Medical Optimization

The target of medical optimization is to identify pre-existing comorbidities and manage them before surgery. Measures to achieve medical optimization in our program are shown in Table 1. It is strongly recommended to stop tobacco and alcohol consumption. Smokers can enroll in a hospital pulmonology program that consists of behavioral support and nicotine replacement therapy, if needed. Pre-operative screening for anemia is evaluated through a blood test in pre-operative assessment. Pre-operative intravenous or oral iron is recommended if hemoglobin levels are $<11 \mathrm{~g} /$ $\mathrm{dL}$. During pre-operative evaluation, other comorbidities such as hypertension, chronic obstructive pulmonary disease, chronic heart disease, and diabetes are managed to gain optimal control before

Table 1 Medical optimization in the prehabilitation program

\begin{tabular}{lll}
\hline & Pre-operative risk & Strategy \\
\hline Tobacco and alcohol & Consumption & $\begin{array}{l}\text { Stop consumption } \\
\text { Hospital pulmonology program based on } \\
\text { nicotine replacement strategy } \\
\text { Intravenous or oral iron intake }\end{array}$ \\
Diabetes & Basal Hb $<11 \mathrm{~g} / \mathrm{dL}$ & $\begin{array}{l}\text { Evaluation and optimization by hospital } \\
\text { endocrinologist }\end{array}$ \\
Chronic disease & HbA1c $>6 \%$ & Pharmacological optimization by \\
& Hypertension, COPD, chronic heart & anesthesiologist or GP \\
Frailty & disease & Geriatrician evaluation \\
Social environment & G-8 score $\leq 14$ points & Personal interview with social assistant \\
& Poor patient's social situation & support to identify individual patient \\
& & situation
\end{tabular}

COPD, chronic obstructive pulmonary disease; GP, general practitioner; Hb, hemoglobin;HbA1c, glycated hemoglobin. 
Table 2 Physical intervention recommended according to $\mathrm{VO}_{2}$ max values in the prehabilitation program

\begin{tabular}{|c|c|c|c|c|c|}
\hline $\begin{array}{l}\text { Group } \\
\left(\mathrm{VO}_{2} \text { max }\right)\end{array}$ & Aerobic exercise & Strength exercise & $\begin{array}{l}\text { Flexibility } \\
\text { exercise }\end{array}$ & IMT exercise & Frequency \\
\hline $\begin{array}{l}\text { Mild } \\
(<12 \mathrm{~mL} / \mathrm{kg} / \mathrm{min})\end{array}$ & $\begin{array}{l}\text { High-intensity interval } \\
\text { walking of } 6 \text { min } \\
\text { (objective }>5000 \\
\text { steps/day) }\end{array}$ & $\begin{array}{l}\text { Wall push-ups } 10 \times 1 \text {, } \\
\text { chair squats } 10 \times 1 \text {, } \\
\text { chair abdominals } \\
10 \times 1\end{array}$ & $\begin{array}{l}\text { Lateral and } \\
\text { frontal arms } \\
\text { movement } \\
10 \times 1\end{array}$ & $\begin{array}{l}\text { Inspiratory threshold- } \\
\text { loading device } \\
\text { 10'/8hours }\end{array}$ & $\begin{array}{l}\text { Daily supervised } \\
\text { physical therapy } \\
\text { program }\end{array}$ \\
\hline $\begin{array}{l}\text { Moderate } \\
(\geq 12-14 \mathrm{~mL} / \mathrm{kg} / \mathrm{min})\end{array}$ & $\begin{array}{l}\text { Walking of } 6 \text { min } \\
\text { rounds at highest } \\
\text { effort } \\
\text { (objective }>7500 \\
\text { steps/day) }\end{array}$ & $\begin{array}{l}\text { Wall push-ups } 10 \times 2 \text {, } \\
\text { chair squats } 10 \times 2 \text {, } \\
\text { chair abdominals } \\
10 \times 2\end{array}$ & $\begin{array}{l}\text { Lateral and } \\
\text { frontal arms } \\
\text { movement } \\
10 \times 2\end{array}$ & $\begin{array}{l}\text { Inspiratory threshold- } \\
\text { loading device } \\
\text { 10'/8hours }\end{array}$ & $\begin{array}{l}\text { Daily home- } \\
\text { based exercise } \\
\text { program }\end{array}$ \\
\hline $\begin{array}{l}\text { Intense (>14 mL/kg/ } \\
\text { min) }\end{array}$ & $\begin{array}{l}\text { Walking of } 6 \mathrm{~min} \\
\text { rounds at highest } \\
\text { effort } \\
\text { (objective }>10000 \\
\text { steps/day) }\end{array}$ & $\begin{array}{l}\text { Wall push-ups } 15 \times 3 \text {, } \\
\text { chair squats } 15 \times 3 \text {, } \\
\text { chair abdominals } \\
15 \times 3 \text { or elastic band }\end{array}$ & $\begin{array}{l}\text { Lateral and } \\
\text { frontal arms } \\
\text { movement } \\
15 \times 3\end{array}$ & $\begin{array}{l}\text { Inspiratory threshold- } \\
\text { loading device } \\
\text { 10'/8 hours }\end{array}$ & $\begin{array}{l}\text { Daily home- } \\
\text { based exercise } \\
\text { program }\end{array}$ \\
\hline
\end{tabular}

IMT, inspiratory muscle training ; $\mathrm{VO}_{2}$ max, maximum oxygen consumption.

surgery. In addition, the G-8 scale, a geriatric screening scale for frailty, is used to evaluate patients older than 70 years. Patients with a positive G-8 score ( $\leq 14$ points) are referred to a geriatrician. In the baseline evaluation we explore the social environment and request individualized support from a social assistant, if necessary.

\section{Physical Intervention}

Functional exercise capacity is evaluated with the 6-MWT test and $\mathrm{VO}_{2}$ max is calculated. Then, an exercise plan is prescribed based on these results (Table 2). The 6-MWT is performed at baseline, before surgery, and 8 weeks after surgery. In addition, all patients are asked to record their physical activity in a diary, and are encouraged to remain as active as possible to continue improving their own post-operative condition after discharge. Patients with $\mathrm{V}_{2} \max <12 \mathrm{~mL} / \mathrm{kg} /$ min undergo a supervised physical therapy program by physiotherapists. Patients with $\mathrm{V}_{2} \max \geq 12 \mathrm{~mL} / \mathrm{kg} / \mathrm{min}$ are further stratified and we prescribe a home-based exercise program consisting of daily training sessions that include aerobic, strength, flexibility, and respiratory exercises at different intensities. Exercise is prescribed following the guidelines of the American College of Sports Medicine. ${ }^{50}$ Additionally, all patients are prescribed inspiratory exercise through inspiratory muscle training. This consists of 10 min sessions every 8 hours using an inspiratory threshold-loading device with maximal inspiratory muscle strength. After surgery, patients are encouraged to start in-hospital exercise as soon as cleared for mobilization by the nursing staff as a part of ERAS program. At this point, and during their hospitalization, patients in more serious physical condition are able to review the exercise program with a physical therapist, in order to ensure that they are both confident and comfortable performing the exercises after discharge.

Nutritional Intervention

Nutritional therapy may involve screening tools and pre-operative dietary advice. We evaluate nutritional status with body mass index, laboratory parameters, and MUST score. The MUST score uses three independent parameters to determine the overall risk of malnutrition: 0 -low, 1-medium, and $\geq 2$ high risk of malnutrition. In addition to the MUST score, serum albumin is assessed at baseline, pre-operative, and at 8 weeks after surgery. Depending on the baseline nutritional parameters, we recommend oral nutritional supplements. In severely malnourished patients (MUST $\geq 2$ or albumin $<3 \mathrm{~g} / \mathrm{dL}$ ), a dietitian creates an individual dietary plan (Table 3). All patients receive a nutritional education program involving food selection and meal planning patterns including a list of home-made recipes of protein supplements adapted to diabetic patients, if necessary. All patients are instructed to take these oral protein supplements daily $30 \mathrm{~min}$ after exercise training to enhance muscle hypertrophy. In addition, all patients receive $50 \mathrm{~g}$ of a carbohydrate-loaded drink 2 hours before surgery and the anesthesiologist prevents their dehydration during surgery by following ERAS guidelines. After surgery, patients are encouraged to start in-hospital oral feeding as soon as possible. All patients receive oral protein supplements during their hospitalization. A dietitian re-assesses malnourished patients during their hospital stay and recommends a dietary plan during the hospitalization and at discharge. All patients are asked to keep a daily diary of food intake to evaluate their compliance with nutritional recommendations.

Table 3 Nutritional intervention according to the MUST score and serum albumin levels in the prehabilitation program

\begin{tabular}{ll}
\hline Parameter & Strategy \\
\hline $\begin{array}{l}\text { MUST score }<2 \\
\text { and/or } \\
\text { albumin } \geq 3 \mathrm{~g} / \mathrm{dL}\end{array}$ & $\begin{array}{l}\text { Dietary recommendation } \\
\text { Increase overall nutritional intake } \\
\text { with oral supplements, like protein } \\
\text { supplementation } 30 \text { min following } \\
\text { training to enhance muscle hypertrophy }\end{array}$ \\
MUST $\geq 2$ and/ & $\begin{array}{l}\text { Refer to dietitian to get a personalized } \\
\text { dietary plan }\end{array}$ \\
or & $\begin{array}{l}\text { Increase overall nutritional intake } \\
\text { albumin }<3 \mathrm{~g} / \mathrm{dL} \\
\text { with oral supplements, like protein } \\
\text { supplementation } 30 \text { min following } \\
\text { training to enhance muscle hypertrophy }\end{array}$
\end{tabular}

MUST, Malnutrition Universal Screening Tool. 
Table 4 Physiological intervention according to the HADS scale in the prehabilitation program

\begin{tabular}{ll}
\hline Parameter & Strategy \\
\hline HADS & Daily home-based relaxation and breathing \\
score $\leq 7$ & exercise after lunch and before bedtime \\
& Supervised group mindfulness hospital \\
& sessions once a week \\
HADS & Personalized expert psychotherapy \\
score $>7$ & Supervised group mindfulness hospital \\
& sessions once a week
\end{tabular}

HADS, Hospital Anxiety and Depression Scale

Psychological Intervention

The psychological status of patients is assessed through the HADS screening test which includes anxiety and depression subscales. Each scale has seven items scored 0 to 3 , and the total score ranges from 0 to 21, with higher scores indicating worse affective or mood disorders. The HADS test is performed at baseline, before surgery, and at 8 weeks after surgery. Patients whose HADS score is $\leq 7$ are prescribed anxiety reduction techniques such as relaxation and breathing exercises, to be performed at home for $20 \mathrm{~min}$ after lunch and before going to bed. Patients whose score is $>7$ are referred to a psychologist, who prescribes personalized treatment and techniques to alleviate pre-operative anxiety or depression symptoms. A psychologist supports these patients during their hospital stay and at discharge (Table 4). In addition, once a week all patients are encouraged to attend a free supervised session of mindfulness at the hospital.

\section{Algorithm of the Prehabilitation Program}

A summary of our prehabilitation program is presented in Figure 2.

A multidisciplinary team of gynecologists, anesthesiologists, physiotherapists, dieticians, psychologists, and geriatricians provides this intervention. We implemented the prehabilitation program in the Department of Gynecologic Oncology at Hospital del Mar in January 2018, and we are currently awaiting the preliminary results of the intervention on patient outcomes.

\section{CONCLUSIONS}

Prehabilitation programs are aimed to optimize patients' functional capacity before an upcoming stressor such as surgery. Emerging data suggest that multimodal prehabilitation programs in major

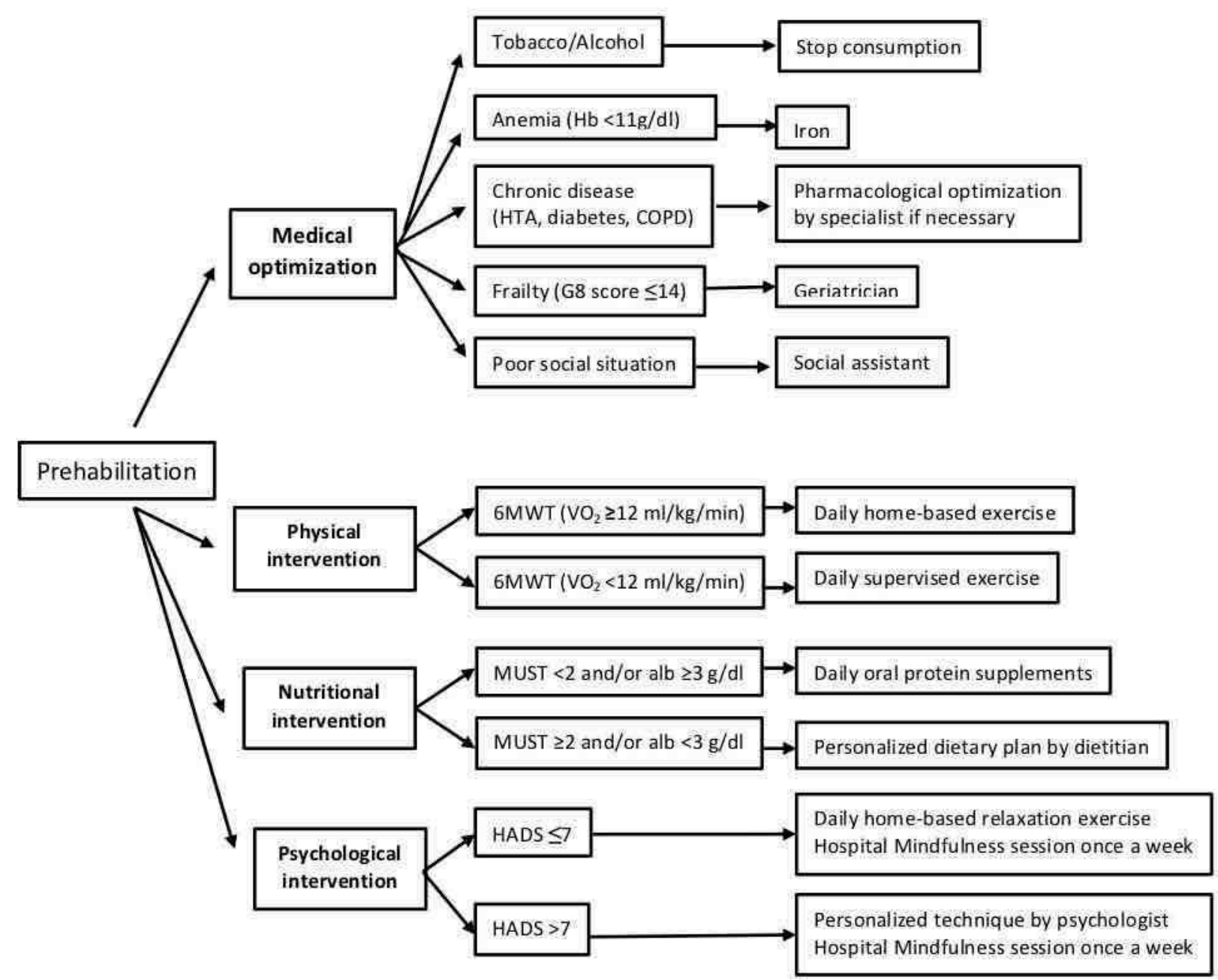

Figure 2 Algorithm of the prehabilitation program for gynecology oncologic patients. alb, albumin; COPD, chronic obstructive pulmonary disease; HADS, Hospital Anxiety and Depression Scale; Hb, hemoglobin; HTA, hypertension; MUST, Malnutrition Universal Screening Tool; $\mathrm{VO}_{2}$, oxygen consumption; 6MWT, 6-min walk test. 


\section{Review Article}

cancer surgeries show a positive impact on patient outcomes, although studies are heterogeneous and high-level evidence to support this is still lacking. Besides, multimodal programs that include medical optimization and physical, nutritional, and psychological interventions show greater positive impact on functional recovery compared with single prehabilitation modality. Therefore, the addition of a multimodal prehabilitation program at diagnosis to an ERAS pathway at pre-operative time might allow complementary efforts and translate into functional improvements for the patient. We therefore suggest a multimodal prehabilitation program based on ERAS guidelines for patients undergoing gynecologic oncology surgery. Nevertheless, new studies are still needed to assess the efficacy of surgical prehabilitation and to standardize protocols in patients of different disciplines, specifically in gynecologic oncology patients.

Acknowledgements The authors thank Alba Gomez, PhD and Alba Llopis, PhD for providing medical writing and editorial support.

Contributors EM, MC and GM conceived the idea for the study. EM, JMS and GM established the methodology. EM and SG wrote the article and conducted the literature examination. All authors were responsible for the writing, critical review, and final approval of the manuscript.

Funding The authors have not declared a specific grant for this research from any funding agency in the public, commercial or not-for-profit sectors.

Competing interests None declared.

Patient consent for publication Not required.

Provenance and peer review Not commissioned; externally peer reviewed.

\section{REFERENCES}

1. Gillis $\mathrm{C}$, Carli F. Promoting perioperative metabolic and nutritional care. Anesthesiology 2015;123:1455-72.

2. Christensen $\mathrm{T}$, Bendix $\mathrm{T}$, Kehlet $\mathrm{H}$. Fatigue and cardiorespiratory function following abdominal surgery. Br J Surg 1982;69:417-9.

3. Kehlet H. Multimodal approach to control postoperative pathophysiology and rehabilitation. Br J Anaesth 1997;78:606-17.

4. Fearon $\mathrm{KCH}$, Ljungqvist $\mathrm{O}$, Von Meyenfeldt $\mathrm{M}$, et al. Enhanced recovery after surgery: a consensus review of clinical care for patients undergoing colonic resection. Clin Nutr 2005;24:466-77.

5. Miralpeix E, Nick AM, Meyer LA, et al. A call for new standard of care in perioperative gynecologic oncology practice: impact of enhanced recovery after surgery (ERAS) programs. Gynecol Oncol 2016;141:371-8

6. Nelson G, Bakkum-Gamez J, Kalogera E, et al. Guidelines for perioperative care in gynecologic/oncology: Enhanced Recovery After Surgery (ERAS) Society recommendations -2019 update. Int $J$ Gynecol Cancer 2019;29:651-68.

7. Li C, Carli F, Lee L, et al. Impact of a trimodal prehabilitation program on functional recovery after colorectal cancer surgery: a pilot study. Surg Endosc 2013;27:1072-82.

8. Sweitzer BJ. Preoperative screening, evaluation, and optimization of the patient's medical status before outpatient surgery. Curr Opin Anaesthesiol 2008;21:711-8.

9. Merki-Künzli C, Kerstan-Huber M, Switalla D, et al. Assessing the value of prehabilitation in patients undergoing colorectal surgery according to the enhanced recovery after surgery (ERAS) pathway for the improvement of postoperative outcomes: protocol for a randomized controlled trial. JMIR Res Protoc 2017;6:e199.

10. Bousquet-Dion G, Awasthi R, Loiselle Sarah-Ève, et al. Evaluation of supervised multimodal prehabilitation programme in cancer patients undergoing colorectal resection: a randomized control trial. Acta Oncol 2018;57:849-59.

11. Gillis C, Loiselle S-E, Fiore JF, et al. Prehabilitation with whey protein supplementation on perioperative functional exercise capacity in patients undergoing colorectal resection for cancer: a pilot doubleblinded randomized placebo-controlled trial. J Acad Nutr Diet 2016;116:802-12.

12. Wilson RJT, Davies S, Yates D, et al. Impaired functional capacity is associated with all-cause mortality after major elective intraabdominal surgery. Br J Anaesth 2010;105:297-303.
13. Carli F, Zavorsky GS. Optimizing functional exercise capacity in the elderly surgical population. Curr Opin Clin Nutr Metab Care 2005;8:23-32.

14. Luther A, Gabriel J, Watson RP, et al. The impact of total body prehabilitation on post-operative outcomes after major abdominal surgery: a systematic review. World J Surg 2018;42:2781-91.

15. Barberan-Garcia A, Ubré M, Roca J, et al. Personalised prehabilitation in high-risk patients undergoing elective major abdominal surgery: a randomized blinded controlled trial. Ann Surg 2018;267:50-6.

16. Dronkers JJ, Chorus AMJ, van Meeteren NLU, et al. The association of pre-operative physical fitness and physical activity with outcome after scheduled major abdominal surgery. Anaesthesia 2013;68:67-73.

17. Moran J, Guinan E, McCormick P, et al. The ability of prehabilitation to influence postoperative outcome after intra-abdominal operation: a systematic review and meta-analysis. Surgery 2016;160:1189-201.

18. Levett DZH, Edwards M, Grocott M, et al. Preparing the patient for surgery to improve outcomes. Best Pract Res Clin Anaesthesiol 2016;30:145-57.

19. Sinclair RCF, Batterham AM, Davies S, et al. Validity of the 6 min walk test in prediction of the anaerobic threshold before major noncardiac surgery. Br J Anaesth 2012;108:30-5.

20. Keeratichananont W, Thanadetsuntorn C, Keeratichananont S. Value of preoperative 6-minute walk test for predicting postoperative pulmonary complications. Ther Adv Respir Dis 2016;10:18-25.

21. Lee L, Schwartzman K, Carli F, et al. The association of the distance walked in 6 min with pre-operative peak oxygen consumption and complications 1 month after colorectal resection. Anaesthesia 2013;68:811-6.

22. Rasekaba T, Lee AL, Naughton MT, et al. The six-minute walk test: a useful metric for the cardiopulmonary patient. Intern Med J 2009;39:495-501.

23. Soares SMdeTP, Nucci LB, da Silva MMdeC, et al. Pulmonary function and physical performance outcomes with preoperative physical therapy in upper abdominal surgery: a randomized controlled trial. Clin Rehabil 2013;27:616-27.

24. Carli F, Charlebois $P$, Stein B, et al. Randomized clinical trial of prehabilitation in colorectal surgery. Br J Surg 2010;97:1187-97.

25. Gillis C, Li C, Lee L, et al. Prehabilitation versus rehabilitation: a randomized control trial in patients undergoing colorectal resection for cancer. Anesthesiology 2014;121:937-47.

26. Hulzebos EHJ, Helders PJM, Favié NJ, et al. Preoperative intensive inspiratory muscle training to prevent postoperative pulmonary complications in high-risk patients undergoing CABG surgery. JAMA 2006;296:1851-7.

27. Hoogeboom TJ, Dronkers JJ, Hulzebos EHJ, et al. Merits of exercise therapy before and after major surgery. Curr Opin Anaesthesiol 2014;27:161-6.

28. Alaparthi GK, Augustine AJ, Anand R, et al. Comparison of diaphragmatic breathing exercise, volume and flow incentive spirometry, on diaphragm excursion and pulmonary function in patients undergoing laparoscopic surgery: a randomized controlled trial. Minim Invasive Surg 2016;2016:1-12.

29. Awasthi R. Multimodal prehabilitation in patients undergoing colorectal cancer resection: the impact of supervised structured perioperative exercise on postoperative functional capacity. master thesis. Concordia University, 2016. Available: https://spectrum. library.concordia.ca/981003/

30. Smith MD, Mccall J, Plank L, et al. Preoperative carbohydrate treatment for enhancing recovery after elective surgery. Cochrane Database Syst Rev 2014;14.

31. Evans DC, Martindale RG, Kiraly LN, et al. Nutrition optimization prior to surgery. Nutr Clin Pract 2014;29:10-21.

32. Ryan AM, Power DG, Daly L, et al. Cancer-associated malnutrition, cachexia and sarcopenia: the skeleton in the hospital closet 40 years later. Proc Nutr Soc 2016;75:199-211.

33. Jie B, Jiang Z-M, Nolan MT, et al. Impact of preoperative nutritional support on clinical outcome in abdominal surgical patients at nutritional risk. Nutrition 2012;28:1022-7.

34. Burden ST, Hill J, Shaffer JL, et al. An unblinded randomised controlled trial of preoperative oral supplements in colorectal cancer patients. J Hum Nutr Diet 2011;24:441-8.

35. Kabata P, Jastrzębski T, Kąkol M, et al. Preoperative nutritional support in cancer patients with no clinical signs of malnutritionprospective randomized controlled trial. Support Care Cancer 2015;23:365-70.

36. Skipper A, Ferguson M, Thompson K, et al. Nutrition screening tools: an analysis of the evidence. JPEN J Parenter Enteral Nutr 2012;36:292-8. 
Review Article

37. Burd NA, Yang Y, Moore DR, et al. Greater stimulation of myofibrillar protein synthesis with ingestion of whey protein isolate $v$. micellar casein at rest and after resistance exercise in elderly men. Br J Nutr 2012;108:958-62.

38. Mavros MN, Athanasiou S, Gkegkes ID, et al. Do psychological variables affect early surgical recovery? PLoS One 2011;6:e20306.

39. $\mathrm{PM} \mathrm{H}$, Masoudi FA, Spertus JA, et al. Depression predicts mortality following cardiac valve surgery. Ann Thorac Surg 2005;79:1255-9.

40. Zigmond AS, Snaith RP. The hospital anxiety and depression scale. Acta Psychiatr Scand 1983;67:361-70.

41. Powell R, Scott NW, Manyande A, et al. Psychological preparation and postoperative outcomes for adults undergoing surgery under general anaesthesia. Cochrane Database Syst Rev 2016;5.

42. Rimer J, Dwan K, Lawlor DA, et al. Exercise for depression. Cochrane Database Syst Rev 2013.

43. Mayo NE, Feldman L, Scott S, et al. Impact of preoperative change in physical function on postoperative recovery: argument supporting prehabilitation for colorectal surgery. Surgery 2011;150:505-14

44. Minnella EM, Bousquet-Dion G, Awasthi R, et al. Multimodal prehabilitation improves functional capacity before and after colorectal surgery for cancer: a five-year research experience. Acta Oncol 2017;56:295-300.
45. Ebner F, Schulz SVW, de Gregorio A, et al. Prehabilitation in gynecological surgery? What do gynecologists know and need to know. Arch Gynecol Obstet 2018;297:27-31.

46. Carli F, Brown R, Kennepohl S. Prehabilitation to enhance postoperative recovery for an octogenarian following roboticassisted hysterectomy with endometrial cancer. J Can Anesth 2012;59:779-84.

47. Vonk Noordegraaf A, Anema JR, van Mechelen W, et al. A personalised eHealth programme reduces the duration until return to work after gynaecological surgery: results of a multicentre randomised trial. Int J Obstet Gy 2014;121:1127-36.

48. Hawkes AL, Quinn M, Gebski V, et al. Improving treatment for obese women with early stage cancer of the uterus: rationale and design of the levonorgestrel intrauterine device \pm metformin \pm weight loss in endometrial cancer (feMME) trial. Contemp Clin Trials 2014;39:14-21.

49. Schmidt S, Vilagut G, Garin O, et al. Reference guidelines for the 12-Item Short-Form Health Survey version 2 based on the Catalan general population. Med Clin 2012;139:613-25.

50. Thompson PD, Arena R, Riebe D, et al. ACSM's New Preparticipation Health Screening Recommendations from ACSM's Guidelines for Exercise Testing and Prescription, Ninth Edition. Curr Sports Med Rep 2013;12:215-7. 